\title{
Robert Sauzet, Les Cévennes catholiques. Histoire d'une fidélité (XVIe-XXe siècle),
}

\section{Brigitte Maillard}

\section{(2) OpenEdition \\ 1 Journals}

Édition électronique

URL : http://journals.openedition.org/abpo/1488

DOI : $10.4000 / a b p o .1488$

ISBN : 978-2-7535-1489-8

ISSN : $2108-6443$

\section{Éditeur}

Presses universitaires de Rennes

\section{Édition imprimée}

Date de publication : 20 mars 2003

Pagination : 170-172

ISBN : 978-2-86847-811-5

ISSN : 0399-0826

\section{Référence électronique}

Brigitte Maillard, « Robert Sauzet, Les Cévennes catholiques. Histoire d'une fidélité (XVle-XXe siècle), », Annales de Bretagne et des Pays de l'Ouest [En ligne], 110-1 | 2003, mis en ligne le 20 mars 2005, consulté le 23 septembre 2020. URL : http://journals.openedition.org/abpo/1488 ; DOI : https:// doi.org/10.4000/abpo.1488 
de l'ouest sur la guerre terrestre et l'humanisent en quelque sorte, il n'en est rien dans les conflits sur mer et dans les colonies, qui restent beaucoup plus âpres. Le principe des armées professionnelles triomphe même s'il est contesté au nom de l'amour de la patrie, sentiment que l'on pense inconnu de cette catégorie de troupes. Mais le problème du recrutement des armées de terre et surtout des équipages n'est pas résolu : la "main d'œuvre " reste rare ou difficile à conserver. À cette date l'armée prussienne fait le plus souvent figure de modèle : contrairement à bien d'autres, elle voit ses effectifs augmenter, grâce en particulier au nombre important de conscrits, et elle présente des traits d'homogénéité que l'armée française ne possède pas, traversée qu'elle est par de graves conflits sociaux dans lesquels s'exprime nettement le malaise ressenti par les familles pauvres de la noblesse ancienne. En France en particulier, la troupe est appelée à assurer des tâches de maintien de l'ordre, en complément de l'activité de la maréchaussée, ou à participer à des travaux d'utilité publique; des rapports d'un type nouveau, faisant une place à une réelle sympathie de la part de la population, se développent avec cette dernière; en 1789, l'armée est largement pénétrée par les idées nouvelles et ne constitue pas un soutien sûr pour le roi.

La dernière partie permet de faire le point sur les questions en débat, les thèmes d'actualité historique, les chantiers ouverts. Les pages que J. Chagniot consacre à l'étude sociale de l'armée française sont nourries de ses connaissances sur la société française, acquises pour la rédaction de sa thèse sur Paris et l'armée au XVIII siècle; ainsi par exemple, les arcanes juridiques de la condition militaire n'ont plus de secret pour lui. On peut y lire aussi un exposé des positions de différents historiens européens sur l'existence d'une "Révolution militaire des temps moderne ", idée défendue par M. Roberts et, avec quelques divergences, par G. Parker, mais sur laquelle J. Chagniot est plus dubitatif.

Écrites avec clarté et élégance, ces pages abordent donc l'histoire militaire sous de très nombreux angles; elles sont fondées sur d'abondantes lectures et une connaissance solide des réalités de la période moderne; elles reflètent la grande familiarité de leur auteur avec tout l'Ancien Régime. Elles participent aussi à l'œuvre entreprise pour la remise à l'honneur de l'histoire militaire, victime du désintérêt pour l'histoire dite " événementielle ". Cet ouvrage se révèle donc d'une grande utilité en particulier pour l'historien non spécialiste mais aussi pour le lecteur non historien qui cherche à comprendre les transformations connues par les armées avant le XIX ${ }^{\mathrm{e}}$ siècle et, plus encore, les rapports entre les régimes politiques, leurs forces armées et les sociétés.

Brigitte MAILLARD

SAUZET, Robert, Les Cévennes catholiques. Histoire d'une fidélité (XVT-XXe siècle), Paris, Perrin, " Pour l'Histoire ", 2002, 415 p.

Le titre donné par R. Sauzet à l'ouvrage qu'il vient de consacrer aux Cévennes montre clairement ses intentions : il entendait mettre au jour des pans encore inconnus, au début du XXI ${ }^{e}$ siècle, du passé de ces montagnes. Sans doute ces pages se fondent-elles sur une approche savante permise par une longue fréquentation des archives mais bien plus encore sur une expérience vécue, une familiarité profonde avec ce pays qui est sa terre natale. Il s'est attaché à mettre au jour l'histoire d'une minorité dont l'existence a été oubliée, et même occul- 
tée par le développement d'une vision unilatérale des événements et par l'importance accordée à l'épisode de la guerre des Camisards; celle-ci, érigée par certains en véritable mythe, a fait disparaître de la mémoire collective l'existence de ces ruraux qui, restés fidèles à la foi de leurs ancêtres, représentent pourtant un bon quart de la population. Au delà même, plus qu'à l'histoire des institutions, il s'est attaché à celle de ces fidèles dont l'existence, quelle que soit l'appartenance religieuse, a été marquée par la dureté de la nature, la violence des hommes et l'opiniâtreté des croyants. Il a voulu faire sortir de l'ombre tout ce petit peuple de paysans, d'artisans, de journaliers, ces dix mille papistes obstinés, dont quelques-uns furent ses ancêtres; la lutte pour la survie dans de difficiles conditions d'existence aurait dû unir ces populations mais la division religieuse entre catholiques et huguenots a été plus forte.

R. Sauzet prouve l'attachement opiniâtre à la foi catholique d'une minorité non négligeable de villages, dont certains parmi les plus isolés des Cévennes, comme son village de Saint-Martial dont l'unanimisme catholique lui a longtemps fait ignorer que les Cévenols étaient en majorité calvinistes. Dans sa jeunesse, il y a connu toutes les manifestations identitaires du catholicisme, héritées de l'Ancien Régime et du XIX ${ }^{\mathrm{e}}$ siècle, que révèlent différentes pratiques : processions, plantations de croix, sonneries de cloches, culte du Christ, de la Vierge et des saints (dont en particulier, celui de saint Blaise, protecteur des cardeurs), testaments, prières pour les morts, et il s'interroge sur les avatars de ces comportements à partir du Xvi ${ }^{\mathrm{e}}$ siècle. Ces ruraux ont montré une capacité de résistance peu commune à la pénétration des idées "nouvelles " au $\mathrm{XVI}^{\mathrm{e}}$ siècle mais aussi à l'acculturation religieuse développée par la Réforme catholique, dont l'austérité n'a rien à envier à la Réforme calviniste. Les calvinistes ont, de leur côté aussi, mis longtemps à adopter les nouveaux comportements de vie, qui bannissaient les réjouissances comme la danse; pour autant ils se sont révélés insensibles aux entreprises des missionnaires qui, au long des siècles, ont échoué dans leur œuvre de conversion, si bien que les " frontières " semblent presque figées dès la fin du Xvi ${ }^{\mathrm{e}}$ siècle. Au XVII siècle, le catholicisme n'a pas réussi à tirer parti de conditions apparemment plus favorables, - la présence de ce bastion de fidélité -, pour réaliser une reconquête. Alors qu'elles furent peu touchées par la guerre contre l'ennemi extérieur, au cours des deux épisodes de guerre civile (à la fin du XVI ${ }^{\mathrm{e}}$ et au début du XVIII ${ }^{\mathrm{e}}$ siècle) qui les ont ravagées, ces montagnes ont subi les manifestations de la force armée sous leurs différentes formes et elles ont aussi pris leur part dans le développement de la violence; sans les occulter, R. Sauzet évoque rapidement les événements des guerres de religion ou de la révolte des camisards pour mieux mettre en évidence des épisodes moins connus, comme ceux des guerres de Rohan, ou démontrer le rôle des "milices". Surgissent aussi de l'oubli quelques fortes personnalités, comme "Sans Peur ", ce curé réfractaire, "vrai " prêtre mais aussi chef de bande pendant la Révolution... Les ruraux, catholiques ou protestants, ont trouvé dans la pratique religieuse les ressources spirituelles nécessaires pour triompher de l'angoisse : les "malheurs des temps" ont pris dans cette région, des caractères parfois extrêmes, la violence répondant à la violence d'où qu'elle vînt, et les exactions fiscales de la monarchie n'ont épargné personne.

Dans ce passé se sont affirmées des oppositions religieuses qui fondent durablement des identités différentes; s'y enracine le dimorphisme politique et religieux qui caractérise l'espace chronologique qui s'étend de la Révolution à la première guerre mondiale; cette dernière a d'ailleurs réuni dans un même sacrifice au service de la patrie Cévenols catholiques et protestants qui ont payé un lourd tribut démographique. Les lecteurs de l'Ouest de la France retrouveront 
dans ces pages bien des points communs avec leur histoire. Cette «terre blanche " est restée longtemps fidèle à la monarchie, alors que les protestants adoptaient plus facilement la République, si bien que les identités confessionnelles et politiques se recouvrent; le vote y est encore majoritairement conservateur. La constitution civile du clergé y a été mal accueillie même si une forte minorité du clergé a prêté le serment; une "Vendée " cévenole a vu le jour avant même que ne se développent les troubles dans les Mauges angevines et le bas Poitou; l'ultramontanisme y est resté longtemps vivant, les heurts à propos de l'école confessionnelle y furent nombreux. En 1906, l'application de la loi de séparation de l'Église et de l'État et la réalisation des "inventaires " nécessitèrent le recours à la force et provoquèrent de violents incidents. L'évolution religieuse est décalée par rapport à celle du reste de la France : les Cévennes catholiques sont restées longtemps, même chez les hommes, une terre de forte pratique religieuse. L'espace y est fortement sacralisé; le " paysage visuel "y est marqué par les nombreuses croix plantées à toutes les époques et le son des cloches est un élément essentiel du "paysage sonore "; les changements dus en partie à la désertification sont récents.

À travers cette étude c'est toute la vie de l'Église catholique qui apparaît, avec ses problèmes, la lenteur de la transformation du clergé, les difficultés de l'acculturation religieuse selon les canons du concile de Trente. Mais c'est aussi un rappel de l'insondable mystère de la foi : comment pouvait-on être catholique dans les montagnes cévenoles? Pourquoi ces ruraux se sont-ils montrés opiniâtres dans la foi catholique alors que leurs voisins, parfois très proches, se convertissaient? Certes tout un ensemble de facteurs " naturels" ou humains peut être évoqué mais il ne suffit pas à expliquer ces choix qui relèvent de la liberté humaine.

Sans jamais abandonner les exigences de l'honnêteté historique, dans ces pages pleines d'empathie pour ces êtres qui furent ses ancêtres mais aussi pour leurs " frères ennemis ", R. Sauzet retrace ainsi l'histoire chaotique d'une conquête de la tolérance, de l'acceptation du vivre ensemble, dont cette région a fait l'expérience dès le XVII et surtout au XVIII ${ }^{e}$ siècle; il nous livre aussi quelques " clés " pour comprendre une situation et des comportements contemporains.

Brigitte MAILLARD

DuPuY, Roger, La politique du peuple XVII - -XXe siècles. Essai, Paris, Albin Michel Histoire, 2002, 255 pages.

Roger Dupuy, quittant les chemins bien balisés d'une chouannerie dont il a exploré toutes les facettes, propose dans cette Politique du peuple, un essai historiographique particulièrement suggestif. Il tente de mettre en perspective plus de deux siècles de l'histoire des mouvements " populaires " en France avec le concept du " populisme ", dont la naissance est classiquement rapportée à la crise boulangiste des années 1880. Il s'agit de croiser de façon permanente " une politique du peuple " - souvent niée ou simplement minorée dans ses aspects " authentiques " et " autonomes " - et des " politiques à destination du peuple ", qui répercutent à des fins plus ou moins avouables certaines revendications populaires : bonapartisme, boulangisme, maréchalisme, voire monarchisme et gaullisme! Comme le raccordement suggéré des deux niveaux n'est pas évident, Roger Dupuy consacre la plus grande partie de l'ouvrage à la Révolution fran- 